\title{
Resolving Band-Structure Evolution and Defect-Induced States of Single Conjugated Oligomers by Scanning Tunneling Microscopy and Tight-Binding Calculations
}

\author{
Shiyong Wang, Weihua Wang, and Nian Lin* \\ Department of Physics, The Hong Kong University of Science and Technology, Hong Kong, China
}

(Received 25 March 2011; published 16 May 2011)

\begin{abstract}
We study single conjugated polyphenylene oligomers consisting of $3 n(2 \leq n \leq 12)$ phenyl units by means of cryogenic scanning tunneling microscopy and spectroscopy. The spatially resolved local densities of states reveal a progressive development of a continuous conduction band out of discrete molecular orbitals as the length of the oligomers increases. The experimental results are satisfactorily described by tight-binding calculations which gave a conduction band bandwidth of $4.5 \pm 0.2 \mathrm{eV}$ and a band gap of $3.1 \pm 0.2 \mathrm{eV}$ for an infinitely long polymer. We observed two types of defects, known as conformational torsional angle misfit and metasite kink. Tight-binding as well as density-functional theory model calculations confirm that both types of defects effectively destroy the delocalization.
\end{abstract}

One-dimensional (1D) conjugated polymers (CP) are both interesting scientifically and important for technological applications [1]. Theoretical descriptions of CP follow one of two routes: a delocalized band theory of solid-state physics or a localized molecular orbital approach of quantum chemistry [2-4]. In spite of their different perspectives, both approaches predict that the molecular orbitals of short oligomers develop progressively into a broad band in the long-length limit [5-7]. The bandlike features have been detected experimentally for short oligomers only $[8,9]$. The majority of attempts follow the so-called oligomer approach, in which the characteristics of short oligomers of increasing chain length are extrapolated to infinite polymers $[10,11]$. There are still significant discrepancies among different theories, as well as between theories and experimental results, and many fundamental properties of CP including bandwidth, band gap, and the influence of various structural defects, etc., are under debate [7].

In particular, precise experimental determination of band structure, i.e., the energy-momentum dispersion, of $\mathrm{CP}$ or long oligomers has had limited successes largely due to the difficulty of growing high-purity single crystalline samples [12]. Above all, the existence of defects presents a severe challenge since defects in a 1D system can strongly alter its electronic structure [13]. Thus to be able to measure the electronic structure of a single $\mathrm{CP}$ whose molecular structure, including the details of defects, can be resolved simultaneously is highly desirable. Recently, Repp et al. demonstrated that scanning tunneling microscopy (STM) can provide an unprecedented view of the electronic structures of individual conjugated oligomers [14]. In this Letter we report the conductance band structures of single poly(para-phenylene) (PPP) oligomers determined by cryogenic $(5 \mathrm{~K})$ STM. We examine the energy-momentum dispersion of the PPP oligomers of up to $16 \mathrm{~nm}$ in length (36 phenyl units) and derive the conduction band bandwidth, energy gap, and the effective mass of charge carriers. Furthermore, we demonstrate and discuss how structural defects interrupt the delocalized states.

The PPP oligomers are generated by the covalent linking of 4,4"-dibromo- $p$-terphenyl monomers [cf. Fig. 1(a)]. The monomers were first deposited on a $\mathrm{Cu}(111)$ surface in ultrahigh vacuum conditions. After annealing at $200{ }^{\circ} \mathrm{C}$, the monomers underwent debromination reactions and were linked by $\mathrm{C}-\mathrm{C}$ bonds forming the PPP oligomers. The resulting oligomers consist of $3 n$ phenyl units. Figure 1(b) is a representative STM topograph showing several PPP oligomers. The STM data reveal that the length of the oligomers varies from 2.4 to $16 \mathrm{~nm}$, i.e., $2 \leq n \leq 12$. These oligomers provide us the opportunity to investigate how the electronic structure of an oligomer develops as its length increases. To probe the local densities of states (LDOS), we measured differential conductance $(d I / d V)$ at specific sites of the single oligomers. For example, Fig. 1(c) shows two differential conductance spectra acquired at a central site and an off-central site of an $n=2$ oligomer. The phenyl systems are weakly adsorbed on a $\mathrm{Cu}(111)$ surface and the molecular electronic (a)

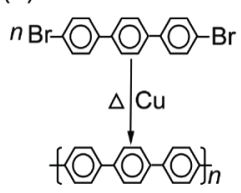

(b)

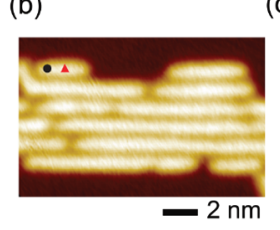

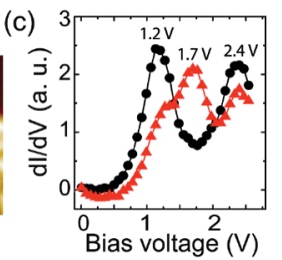

FIG. 1 (color online). (a) Covalent linking of dibromo$p$-terphenyl monomers forming PPP oligomer consisting of $3 n$ phenyl units. (b) STM image showing oligomers with different length. (1.0 V; $0.3 \mathrm{nA})$. (c) $d I / d V$ spectra measured at central and off-central sites of an $n=2$ oligomer as marked in (b). 
states are largely preserved [15]. The $d I / d V$ spectra reflect the intrinsic features of the molecular states.

To identify the origin of these states, we acquired $d I / d V$ spectra at sequential sites along single oligomers. The upper panel of Fig. 2(a) is a spatial distribution of the LDOS plot of an $n=2$ oligomer. One can see that the lowest-energy state at $1.2 \mathrm{~V}$ is distributed across the oligomer with the maximum intensity located in the middle of the oligomer. The $1.7 \mathrm{~V}$ state has two intensity maxima, which are distributed equally in the left half and right half of the oligomer. The $2.4 \mathrm{~V}$ state has three intensity maxima distributed in a symmetrical fashion with the middle maximum located at the middle of the oligomer. The three states were confirmed through density-functional theory (DFT) calculations using the Gaussian software to correspond to the lowest two unoccupied molecular orbitals constructed by the lowest unoccupied molecular orbital (LUMO) of benzene [16]. The LDOS plots of $n=3$ and 4 oligomers, as shown in the upper panels in Figs. 2(b) and 2(c), respectively, exhibit similar states of discrete energy levels but with more modes in the same energy range. In contrast, the longest oligomer of $n=12$ does not show the discrete states but possesses rather states with a continuous bandlike feature [upper panel in Fig. 2(f)]. The states of the oligomers of $n=6$ [upper panel in Fig. 2(d)] and $n=8$ [upper panel in Fig. 2(e)], the intermediate-length oligomers, are somewhere between the discrete levels and the continuous band. We inspected oligomers of other lengths too (i.e., $n=5,7,9,10$, and 11). We found that the oligomers of 24 phenyl units or longer clearly exhibit the bandlike signatures. Figure 2(g) displays the energy levels of the first four unoccupied states as a function of the oligomer length ( $N=3 n$, where $N$ is the number of phenyl units). The energy levels of the short oligomer chains are well separated. As the length of the oligomer increases, the separation between the energy levels decreases. In the limit of long oligomer length, the discrete energy levels merge into a continuous band. The spatially resolved LDOS data as well as the length-dependent energies demonstrate unambiguously, at the single-molecule level, that the electronic states of the PPP oligomers develop progressively from the discrete levels to the continuous band as the length of the oligomers increases.

The total densities of states (DOS), obtained by averaging all the $d I / d V$ spectra taken along single oligomers, also evidence this progressive development of the bandlike feature. As shown in Fig. 2(h), the DOS of the shorter oligomers (cf. $n=2$ or $n=3$ ) show multiple peaks. Such a feature is absent in the longer oligomers (cf. $n=8$ ). On the other hand, the peak at low energy $(\sim 1.0 \mathrm{eV}$, i.e., LUMO level) becomes stronger as the oligomer length increases. The enhancement of this low energy state also signifies the emergence of a 1D band [17].

We carried out tight-binding (TB) calculations to model the PPP oligomers. Since the measurements were conducted in the energy range nearing the Fermi level where only one band was detected, we used a single-band model in the TB calculation. The TB approximation took into account only the nearest-neighbor overlap. The lower panels in Figs. 2(a) to 2(f) show the simulated LDOS plots obtained from the TB calculations. The calculations reproduce the main features of the experimental data fairly well, implying that the discrete levels in the short oligomers expand into a continuous band in the long oligomers in a

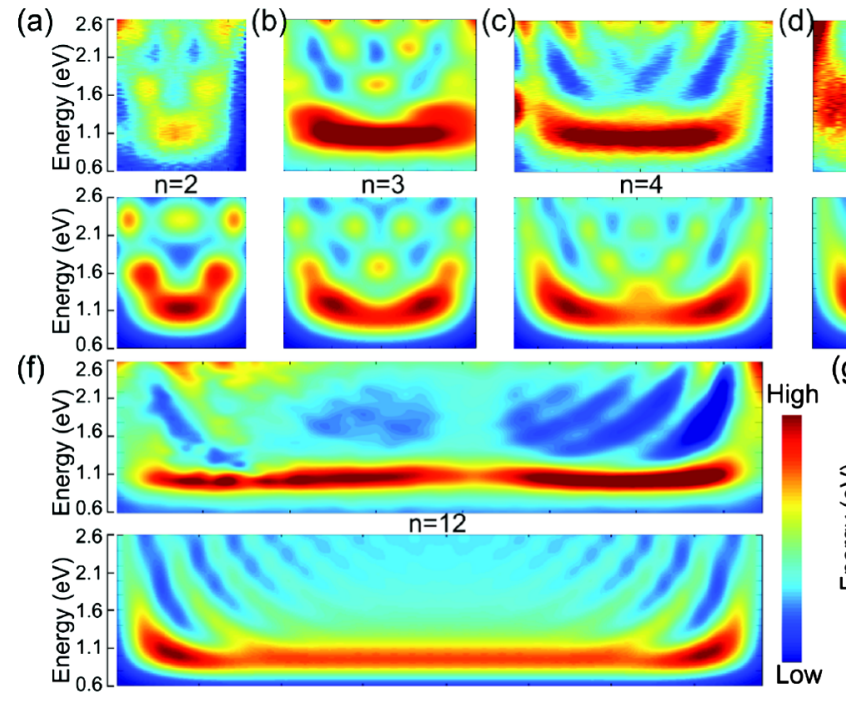

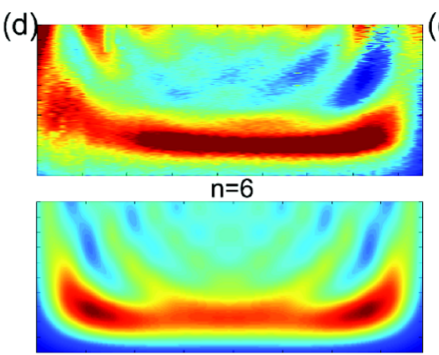

(g)

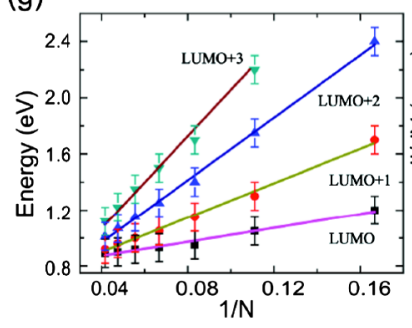

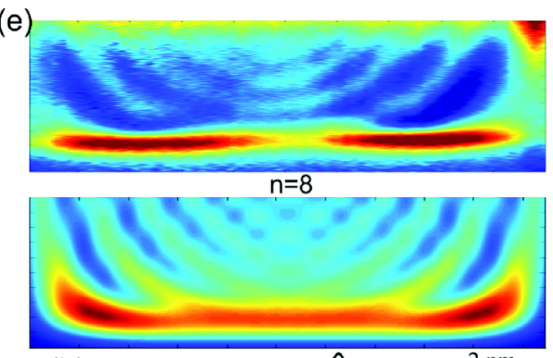

(h)

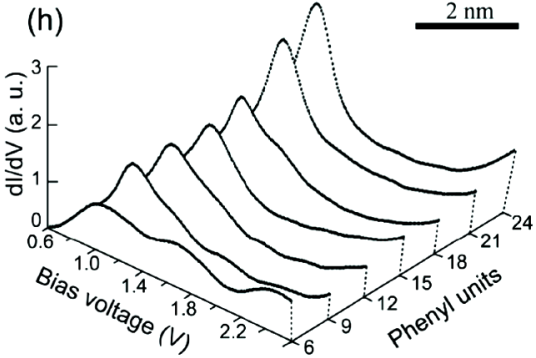

FIG. 2 (color online). (a)-(f) Upper panels: experimental LDOS plots of oligomers with different length $(d I / d V$ spectrum is normalized by $I / V$ ). The vertical axis is energy, the horizontal axis is position along the oligomer, and the color indicates the LDOS intensity. Lower panels: tight-binding simulated LDOS plots. (All the images are plotted in the same length scale.) (g) The four lowest molecular orbital energies (symbols) as a function of oligomer length ( $N=3 n$, where $N$ is the number of phenyl units); solid lines: linear fitting. (h) Total densities of states of oligomers of $n=2$ to 8 . 
way that can be modeled by introducing hopping integrals between nearest-neighbor phenyl units while the single phenyl units retain their molecular orbital signature [5-7].

The LDOS plots allow one to determine the relationship between energy $E$ and wave vector $k\left[k_{m}=m \pi /(\mathrm{Na}), 1<\right.$ $m<N][14,18,19]$. Figure 3(a) displays the $E(k)$ relationships of all states resolved on oligomers of $n=2$ to 10 . The curve in Fig. 3(a) is a $E(k)$ dispersion relationship obtained through TB calculations: $E(k)=\varepsilon-2 t \cos (k a)$, where $a=0.43 \mathrm{~nm}$ is the dimension of a phenyl unit, $\varepsilon=2.9 \mathrm{eV}$ is the on-site energy, and $t=1.0 \mathrm{eV}$ is the nearest-neighbor hopping integral. In general, the experimental data points follow the theoretical curve closely, showing that the PPP oligomers of various lengths can be sufficiently described by the simple TB model. The hopping integral $t$ reflects the nearest-neighbor interaction between adjacent phenyl units. This term depends on the dihedral angle $\theta$ between the adjacent phenyl units as given by $t \propto \cos (\theta)$ [20,21]. We carried out DFT calculations for a PPP oligomer of $n=4$ and found that when the dihedral angle $\theta$ is set to $28^{\circ}$, the DFT calculations can reproduce the experimental $E(k)$ relationship. Hence, we suggest the dihedral angle of the PPP oligomers to be $28^{\circ}$ as they are adsorbed on the $\mathrm{Cu}(111)$ surface. This value is consistent with the previously reported dihedral angle of $20^{\circ}-35^{\circ}$ [20].

Furthermore, we examined the variation of $\varepsilon$ and $t$ in terms of oligomer length. We fitted the experimental data ( $E$ and $k$ ) of the oligomers of a specific length using the TB approximation. Both $\varepsilon$ and $t$ increase for longer oligomers. Since the behaviors of the longer oligomers $(n>8)$ already approach those of an infinitely long polymer, the length-dependent $\varepsilon$ and $t$ allow us to derive reliable bandstructure parameters of infinitely long PPP polymers. First we discuss the conduction band (CB) bandwidth which is defined by $4 t$. Figure $3(\mathrm{~b})$ (triangles) shows that the $\mathrm{CB}$ bandwidth reaches $4.3 \pm 0.2 \mathrm{eV}$ for the longest oligomer measured $(n=10)$ and approaches $4.5 \pm 0.2 \mathrm{eV}$ for an infinitely long PPP polymer via linear extrapolation.
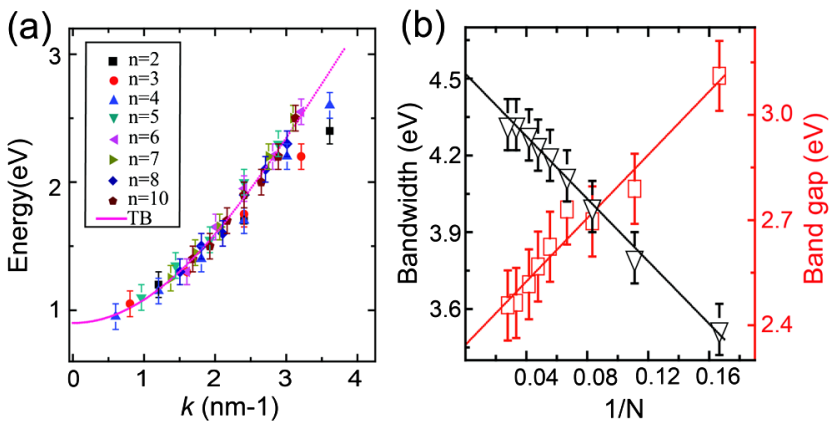

FIG. 3 (color online). (a) Energy-momentum relation of all states resolved on oligomers of $n=2$ to 10 (symbols); solid curve: a tight-binding calculated dispersion. (b) Conduction band bandwidth (triangles) and band gap (squares) as a function of oligomer length; solid lines: linear fitting.
Experimental data of the CB bandwidth of PPP polymers have rarely been reported before. The reported theoretical values, however, span a wide range from $1.46 \mathrm{eV}$ (TBA) to $5.60 \mathrm{eV}$ (HF) $[5,11,20,22-27]$. Considering that the dihedral angle in our case is $28^{\circ}$, the $4.5 \mathrm{eV} \mathrm{CB}$ bandwidth reported here is consistent with the theoretical prediction of Champagne et al. [27].

The band gap cannot be determined directly from our experimental data since the tunneling spectra do not resolve any occupied states. Nevertheless, one can estimate the band gap according to the symmetric characteristics of the $\mathrm{CB}$ and the valence band (VB) $[8,20]$. Assuming the VB is described by $E(k)=-\varepsilon-2 \delta+2 t \cos (k a)$, where $\delta$ defines the shifting of the Fermi level away from the middle of the band gap, the band gap can be expressed as $(\varepsilon+\delta)-4 t \cos \left(\frac{\pi}{N}\right)$. Using the reported band gap value of sexiphenyl $3.9 \mathrm{eV}$ (or $3.1 \mathrm{eV}$ ) [28,29], we found $\delta=0.42 \mathrm{eV}$ (or $0.38 \mathrm{eV}$ ). Figure 3(b) (squares) summarizes the band gap of different oligomers. The gap increases almost linearly with $1 / N$, in agreement with the well-known $1 / N$ law. Using linear extrapolation, the band gap eventually approaches $3.1 \pm 0.2 \mathrm{eV}$ (or $2.3 \pm 0.2 \mathrm{eV}$ ) for an infinitely long PPP polymer. Like CB bandwidth, the band gap value of PPP is also debated in the literature [22,23,30-33]. Our experimental data were acquired by measuring well-defined single oligomers, many uncertainties encountered in other experimental methods, for example, interchain effects or defect influence, can be excluded. Hence our approach can provide more reliable values of the band gap, as well as the $\mathrm{CB}$ bandwidth. Another quantity that can be obtained from the band dispersion is the effective mass $\left(m^{*}\right)$ of charge carriers. Near the CB zone center, $m^{*}=0.23 m_{e}$ for the $n=3$ oligomer, $0.19 m_{e}$ for the $n=10$ oligomer, and $0.18 m_{e}$ for an infinitely long PPP polymer. These values are larger than the theoretical prediction of $0.10 m_{e}$ but, nevertheless, suggest the high carrier mobility in PPP polymers [25].

Many oligomers examined in our experiments have defects. The first type of defect is a point defect which, however, is almost indistinguishable in STM topography. The oligomer in the dashed square in Fig. 4(a) has three such point defects. Figure 4(b) is the LDOS plot of this oligomer, showing the states are strongly disturbed or even entirely interrupted by the defects. The line profile shows there is no sharp discontinuity in oligomer's morphology at these defects. We propose that this type of defect is not due to a break in the oligomer chain structure [cf. the gaps between the lined-up oligomers in Fig. 1(b)], but is rather caused by a conformational torsional angle misfit, i.e., an enlarged dihedral angle between adjacent phenyl units. We simulated this type of defects by reducing $t$ at a specific site of the oligomer in TB calculations. Figure 4(c) shows that while $t$ is reduced at the three defect locations ( $t=0.85,0.85$, and $0.70 \mathrm{eV}$ from left to right) while $t=1.0 \mathrm{eV}$ at rest sites, the simulated LDOS plot matches 


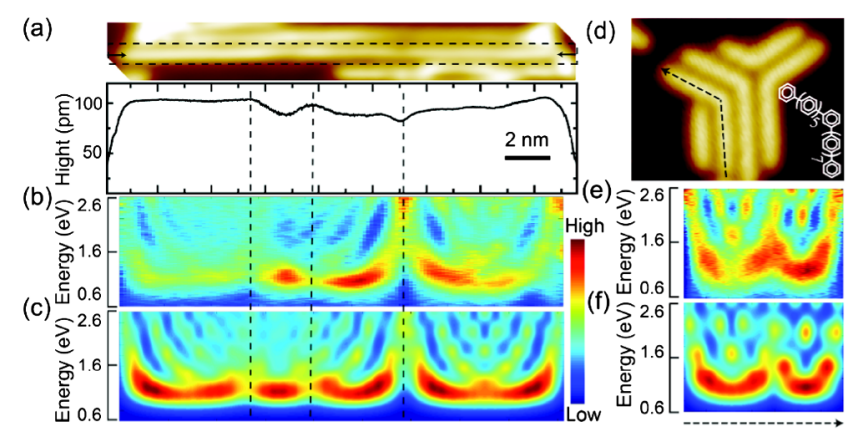

FIG. 4 (color online). (a) Upper panel: STM image showing an $n=13$ oligomer (between two dashed lines) with point defects $(0.8 \mathrm{~V} ; 0.3 \mathrm{nA})$; lower panel: line profile along the oligomer (as marked by the arrows). (b) and (c) Experimentally measured and tight-binding calculated LDOS plots. (d) STM image of chains each containing a $120^{\circ}$ kink $(1.0 \mathrm{~V} ; 0.3 \mathrm{nA})$. (Inset: structural model showing the metasite kink.) (e) and (f) Experimentally measured and tight-binding calculated LDOS plots of the oligomer marked by the dashed line with $n=5$. (All images are on the same scale.)

the experimental one very well. The reduced $t$ of 0.85 or $0.70 \mathrm{eV}$ results in an angle of $41^{\circ}$ or $52^{\circ}$, respectively. To further substantiate the proposed torsional angle misfit mechanism, we carried out DFT calculations for an $n=4$ oligomer which has a misfit dihedral angle of $41^{\circ}$ or $52^{\circ}$ and a dihedral angle of $28^{\circ}$ elsewhere. The results show that the states on either side of the misfit dihedral angle resemble those of an independent short oligomer [16], confirming the experimental data and the TB calculations. This type of defect may originate from the growth processes of oligomers.

Another type of defect is presented in Fig. 4(d), which shows three chains each containing a $120^{\circ}$ kink. At a kink, two oligomers are linked to a hingelike phenyl unit at its meta positions as illustrated by the inset model. Such defects may occur when the bromine atom swaps position with a neighboring hydrogen atom in the growth process. The LDOS plot shown in Fig. 4(e) reveals that the two parts separated by the kink behave as two independent PPP oligomers, suggesting very weak coupling between the two parts. DFT model calculations for an $n=4$ oligomer which contains a $120 \mathrm{kink}^{\circ}$ also reveal that the states of the two parts exhibit the characteristics of two independent oligomers [16]. We simulated this type of defect using TB calculations by setting the hopping integral $t=0.70 \mathrm{eV}$ at the two metasites of the hinge phenyl while $t=1.0 \mathrm{eV}$ at rest sites. The resulting LDOS plot is shown in Fig. 4(f), which nicely reproduces the experimental data. Such defects may significantly modify the transport properties of the polymer.

This work is supported by the Hong Kong RGC (Grant No. 602409). *phnlin@ust.hk

[1] A. J. Heeger, Rev. Mod. Phys. 73, 681 (2001).

[2] N. Kirova, S. Brazovskii, and A. R. Bishop, Synth. Met. 100, 29 (1999).

[3] M. J. Rice and Y. N. Gartstein, Phys. Rev. Lett. 73, 2504 (1994).

[4] C. Ambrosch-Draxl, J. A. Majewski, P. Vogl, and G. Leising, Phys. Rev. B 51, 9668 (1995).

[5] P. Puschnig and C. Ambrosch-Draxl, Phys. Rev. B 60, 7891 (1999).

[6] E. Zojer, Z. Shuai, G. Leising and J. L. Bredas, J. Chem. Phys. 111, 1668 (1999).

[7] R. Telesca et al., Phys. Rev. B 63, 155112 (2001).

[8] E. Zojer et al., Phys. Rev. B 56, 10138 (1997).

[9] G. Koller et al., Science 317, 351 (2007).

[10] A. Niko et al., Synth. Met. 76, 177 (1996).

[11] K. Seki et al., Chem. Phys. 91, 459 (1984).

[12] W. R. Salaneck and J.L. Bredas, Synth. Met. 67, 15 (1994).

[13] A. J. Heeger, S. Kivelson, J. R. Schrieffer and W. P. Su, Rev. Mod. Phys. 60, 781 (1988).

[14] J. Repp, P. Liljeroth, and G. Meyer, Nature Phys. 6, 975 (2010).

[15] P. S. Bagus, K. Hermann and C. Wöll, J. Chem. Phys. 123, 184109 (2005).

[16] See supplemental material at http://link.aps.org/ supplemental/10.1103/PhysRevLett.106.206803 for DFT [Becke three-parameter Lee-Yang-Parr (hybrid functional)/6-31g using GAUSSION03 software] calculated molecular orbitals.

[17] L. Van Hove, Phys. Rev. 89, 1189 (1953).

[18] C. X. Cui, M. Kertesz, and Y. Jiang, J. Phys. Chem. 94, 5172 (1990).

[19] A. Imamura, J. Chem. Phys. 52, 3168 (1970).

[20] M. S. Miao, V.E. Van Doren, P.E. Van Camp, and G. Straub, Comput. Mater. Sci. 10, 362 (1998).

[21] M. Alves-Santos et al., J. Comput. Chem. 27, 217 (2006).

[22] F. Bogar, W. Forner, E. Kapuy, and J. Ladik, J. Mol. Struct., Theochem 391, 193 (1997).

[23] M. S. Miao et al., J. Chem. Phys. 109, 9623 (1998).

[24] J. L. Bredas et al., J. Chem. Phys. 77, 371 (1982).

[25] K. Tanaka et al., Synth. Met. 20, 333 (1987).

[26] M.E. Vaschetto, A.P. Monkman and M. Springborg, J. Mol. Struct., Theochem 468, 181 (1999).

[27] B. Champagne, D. H. Mosley, J.G. Fripiat, and J.M. Andre, Phys. Rev. B 54, 2381 (1996).

[28] W. Graupner et al., Mol. Cryst. Liq. Cryst. 256, 549 (1994).

[29] L.W. Shacklette et al., J. Chem. Phys. 73, 4098 (1980).

[30] G. Grem, G. Leditzky, B. Ullrich and G. Leising, Adv. Mater. 4, 36 (1992).

[31] E. K. Miller, G. S. Maskel, C. Y. Yang, and A. J. Heeger, Phys. Rev. B 60, 8028 (1999).

[32] J. L. Bredas et al., Phys. Rev. B 29, 6761 (1984).

[33] S. J. Yang, P. Olishevski, and M. Kertesz, Synth. Met. 141, 171 (2004). 